\title{
Directed transport in driven optical lattices by gauge generation
}

\author{
C. E. Creffield and F. Sols \\ Departamento de Física de Materiales, Universidad Complutense de Madrid, E-28040, Madrid, Spain
}

(Received 15 March 2011; published 24 August 2011)

\begin{abstract}
We examine the dynamics of ultracold atoms held in optical-lattice potentials. By controlling the switching of a periodic driving potential we show how a phase-induced renormalization of the intersite tunneling can be used to produce directed motion and control wave-packet spreading. We further show how this generation of a synthetic gauge potential can be used to split and recombine wave packets, providing an attractive route to implementing quantum computing tasks.
\end{abstract}

DOI: 10.1103/PhysRevA.84.023630

PACS number(s): 67.85.Hj, 03.65.Vf, 05.60.Gg

\section{INTRODUCTION}

In recent years enormous experimental progress has been made in creating and trapping ultracold atom gases [1]. When placed in an optical-lattice potential these gases provide extremely clean and controllable implementations of interacting lattice systems since parameters such as the interparticle interaction and the lattice depth and spacing are all readily tunable. Dissipation and decoherence effects are typically extremely weak, allowing the quantum coherent behavior of these systems to be directly observed.

In contrast to electronic systems, however, trapped atoms are uncharged, and so electric or magnetic fields cannot easily be used to produce or regulate transport. Due to their excellent coherence properties, one means of controlling the dynamics of the atoms is via quantum interference effects. A notable example is termed "coherent destruction of tunneling" (CDT), in which a periodic driving of the lattice causes the amplitude of the intersite hopping to be renormalized [2]. This renormalization has been seen directly in the expansion of trapped Bose-Einstein condensates [3-5] and has been used very recently to produce the fascinating phenomenon of "super Bloch oscillations" [5,6] and to induce the quantum phase transition [7] between a superfluid and an insulator.

In this paper we show that, as well as controlling the amplitude of the hopping, a periodic driving field can also be used to produce a tunneling phase equivalent to a U(1) gauge potential. This gauge potential arises from the combined effect of the phase of the driving field and the careful control of the switching condition. Although here we only consider one-dimensional lattices, the technique can also be similarly applied to create hopping phases in higher-dimensional systems. In this case the phases can be interpreted as AharonovBohm phases picked up by a particle hopping from site to site, corresponding to a synthetic magnetic field threading the lattice [8]. Other schemes have been devised to produce such gauge potentials in cold-atom systems, including lattice rotations, state-dependent optical potentials [9], or phase imprinting [10]. Our procedure, however, has an appealing simplicity in that it requires only the periodic vibration of the lattice potential, which is easily produced in experiment. We show how the driving can be used to control both the spreading and position of an initial wave packet and, in particular, how a directed current of nondispersing wave packets can be induced. We shall also demonstrate how wave packets can be split, guided, and recombined in a controllable and robust manner that is accessible to current experiments.

\section{MODEL}

A gas of weakly interacting ultracold bosonic atoms can be described well by the Gross-Pitaevskii equation (GPE). When a sufficiently deep optical-lattice potential is applied, the wave function will localize mainly in the potential minima defining the lattice sites, making it convenient to use a discretized form of the GPE:

$$
i \frac{\partial \psi_{j}}{\partial t}=-\left(J \psi_{j+1}+J^{\dagger} \psi_{j-1}\right)+g\left|\psi_{j}\right|^{2} \psi_{j}+j V(t) \psi_{j} .
$$

Here, $\psi_{j}$ denotes the system's wave function on lattice site $j$, and $J$ describes the the tunneling amplitude between nearestneighbor sites. Interactions between the bosons are given by a mean-field interaction, set by the nonlinearity parameter $g$. The time-dependent driving potential is assumed to rise linearly across the lattice $[3,4,6]$, and has a time dependence given by $V(t)=\Delta+K \sin (\omega t+\phi)$, where $\Delta$ is a static tilt of the lattice potential and $\omega$ and $K$ are the frequency and amplitude, respectively, of the oscillating component.

As an initial state we take a Gaussian wave packet, $\psi_{j}=$ $N \exp \left[-j^{2} /\left(2 \sigma_{0}^{2}\right)+i \theta_{j}\right]$, where $\sigma_{0}$ is the initial width of the wave packet measured in units of the lattice spacing and $N$ normalizes the wave function to unity. This choice of initial state mimics the experimental situation $[3,4,6]$, in which the condensate is prepared in a harmonic trap and so typically has a Gaussian profile when transferred to the optical lattice. Note also that we explicitly include a site-dependent phase term $\theta_{j}$ in the wave function.

\section{ANALYSIS}

We first consider the noninteracting case $(g=0)$. The Hamiltonian describing the system (1) is then $T$ periodic in time, where $T=2 \pi / \omega$, and the natural framework to describe its time evolution is Floquet theory. This reveals that, in the high-frequency limit $(\omega>J)$, the time-dependent driven system can be described by an effective static Hamiltonian, whose parameters can be systematically evaluated by using perturbation theory [11] on the Floquet states. While these states are explicitly time-dependent, being $T$-periodic functions, their time variation is rather weak in the high-frequency limit. This is the origin, for example, of the well-known 
negligible time dependence of CDT [2], as compared to the large oscillations observed at low driving frequencies when dynamical localization occurs instead [12].

To first order the tunneling amplitudes are modified as $J \rightarrow J_{\text {eff }}=J\left\langle\exp \left[-i \int_{0}^{t} V\left(t^{\prime}\right) d t^{\prime}\right]\right\rangle$, where $\langle\cdots\rangle$ indicates a time average over the driving period $T$. We restrict ourselves to considering the case of resonant driving, when $\Delta=n \omega$, which yields the result

$$
J_{\mathrm{eff}} / J=e^{-i(K / \omega) \cos \phi} e^{i n(\phi+\pi / 2)} \mathcal{J}_{n}(K / \omega),
$$

where $\mathcal{J}_{n}$ is the $n$th Bessel function of the first kind. For the case of $n=0$, a similar expression was obtained in Ref. [13] for a driven Bose-Hubbard model. There, a ramped driving potential was used to adiabatically transform the ground state of the Hamiltonian to a stroboscopically current-carrying Floquet state. Here, however, we use the tunneling phase in a very different way; namely, to control the nonequilibrium dynamics of an expanding atomic wave packet. This, in conjunction with the weak time dependence of the Floquet states in the high-frequency regime, means that the results we present are not stroboscopic and have a negligible dependence on the moment within each driving period at which the system is measured.

From Eq. (2) we can immediately note the importance of the phase of the driving, $\phi$. For a cosinusoidal driving $(\phi= \pm \pi / 2)$, which is the most frequently considered case in the literature, this result simplifies to yield $J_{\text {eff }} / J=$ $(-1)^{n} \mathcal{J}_{n}(K / \omega)$ - the well-known Bessel function renormalization of tunneling found in CDT. For sinusoidal driving $(\phi=0)$, however, the tunneling additionally acquires a phase $J_{\text {eff }} / J=\exp [-i(K / \omega-n \pi / 2)] \mathcal{J}_{n}(K / \omega)$.

It is natural to ask whether this tunneling phase has physical implications, since it would appear that $\phi$ can simply be eliminated by a shift of the time coordinate. It is important to note, however, that we consider the driving potential $V(t)$ to be switched on at a specific moment $t=0$, in common with experimental implementations [5,6]. This gives the time origin and thus the driving-phase an unambiguous definition and, consequently, $\phi$ can indeed be of experimental relevance, as noted in Ref. [14]. This differs from many theoretical analyses [15], in which the steady-state properties of a driven system are considered and the driving is implicitly assumed to have been turned on at $t \rightarrow-\infty$. In such cases the phase of the driving is indeed unimportant.

The expansion of an initially Gaussian condensate in a periodically driven lattice was analyzed in Ref. [16] for real values of $J_{\text {eff }}$. Extending this analysis to complex $J_{\text {eff }}$ gives the result

$$
\sigma(t)=\sigma_{0} \sqrt{1+\left(\operatorname{Re}\left[J_{\mathrm{eff}}\right] t / \sigma_{0}^{2}\right)^{2}} .
$$

We thus see that the spreading of the wave packet is governed by the real component of $J_{\text {eff }}$. For $n=0$, for example, $\operatorname{Re}\left[J_{\text {eff }}\right]=\mathcal{J}_{0}(K / \omega) \cos [(K / \omega) \cos \phi]$, and so as well as freezing at the "standard" CDT condition (when the Bessel function vanishes), expansion is also suppressed at an additional set of values where $\cos [(K / \omega) \cos \phi]=0$.

As well as the expansion of the condensate, another useful experimental measurement is its center-of-mass motion. In the absence of driving, our system has the standard spectrum of a noninteracting lattice model, $E_{k}=-2 J \cos k$. When the system is driven, we can replace the energies $E_{k}$ with quasienergies, obtained as solutions of the Floquet equation, to obtain the new dispersion relation $\varepsilon_{k}=-2\left|J_{\text {eff }}\right| \cos \left(k-k_{0}\right)$, where $J_{\text {eff }}=\left|J_{\text {eff }}\right| \exp \left[i k_{0}\right]$. The effect of the tunneling phase is thus not to alter the quasienergy spectrum of the system, but to displace the wave packet to another point in the first Brillouin zone. In analogy with the familiar semiclassical expression we can now define a mean group velocity $\bar{v}_{g}=d \varepsilon_{k} / d k$, where the average is taken over one period of the driving, to obtain the final result

$$
\bar{v}_{g}=-2 \operatorname{Im}\left[J_{\mathrm{eff}}\right] .
$$

We thus arrive at the rather elegant result that the two quantities most accessible to experiment-the wave-packet expansion and its center-of-mass motion-are directly related to the real and imaginary parts, respectively, of $J_{\text {eff }}$.

\section{A. Directed transport}

To verify these results we numerically simulate the model (1) for a 200 -site lattice with no static tilt $(\Delta=0)$ and take the onsite phases $\theta_{j}$ to be constant. In Fig. 1(a) we show the condensate's expansion for a cosinusoidal driving for several values of $K / \omega$. These curves consist of an initial quadratic dependence on $t$ followed by a linear ballistic expansion at long times [16] and clearly show how varying $K / \omega$ controls the condensate spreading. Equation (3) can be used to extract the value of $\left|\operatorname{Re}\left[J_{\text {eff }}\right]\right|$ from these expansion curves, which we plot in Fig. 1(b). The expected Bessel-function depen-
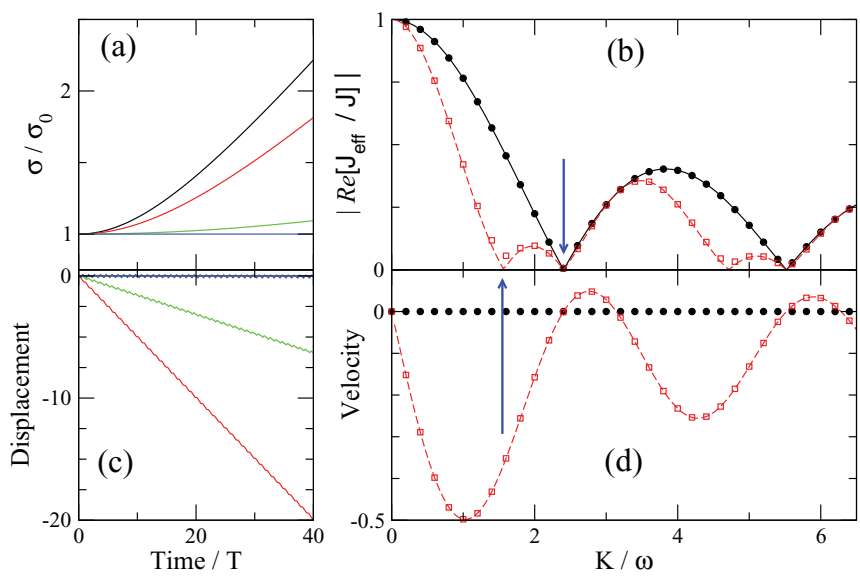

FIG. 1. (Color online) Response of a Gaussian wave packet $\left(\sigma_{0}=4\right)$ to a periodic driving potential, $\omega=16 \mathrm{~J}$. (a) Wave-packet expansion under cosinusoidal driving; from top to bottom $K / \omega=$ $0,1,2,2.4$. For $K / \omega=2.4$, CDT occurs, and the expansion is suppressed. (b) Real component of $J_{\text {eff }}$ extracted from the expansion curves for cosinusoidal (black circles) and sinusoidal (red squares) driving. The curves show the theoretical prediction obtained from Eq. (3). (c) Displacement of a wave packet under sinusoidal driving in units of the lattice spacing. For $K / \omega=0$ and 2.4 no displacement occurs; otherwise, it increases linearly with time. (d) As in (b), for the wave-packet velocity, given in units of $d_{L} / T$ where $d_{L}$ is the lattice spacing. Vertical blue arrows mark the driving parameters $K / \omega=\pi / 2$ (dispersionless directed transport) and $K / \omega=2.404$ (complete suppression of dynamics). 
dence of $J_{\text {eff }}$ is clearly seen, with the condensate expansion being frozen at the zeros of $\mathcal{J}_{0}$ (for $K / \omega=2.40,5.52, \ldots$ ). However, the corresponding expansion for sinusoidal driving shows an additional set of zeros at $K / \omega=\pi / 2,3 \pi / 2, \ldots$, in exact agreement with Eq. (2) for $n=0$. At these values of driving, the suppression of the expansion arises from a very different cause; the tunneling phase displaces the wave packet to $k_{0}=\pi / 2$ in the first Brillouin zone where the quasienergy bands have an inflexion, causing the effective mass to diverge and so quenching the spreading of the wave packet.

In Fig. 1(c) we show the motion of the center of mass of the condensate under sinusoidal driving. The wave packet, initially at rest, begins to move at a constant rate, depending on $K / \omega$. In Fig. 1(d) we plot the velocity corresponding to this displacement, and find that it agrees excellently with the predicted mean group velocity $\bar{v}_{g}$ [Eq. (4)]. Under cosinusoidal driving, however, the velocity of the wave packet is zero, also as predicted.

We note that, to obtain Eqs. (3) and (4), we have assumed that the driving field can be turned on instantaneously. In experiment, of course, this idealized behavior is not possible. To check if our results are robust against this effect, we have included a ramp function in $V(t)$ to describe the effect of turning the field on from zero during a short, but finite, time interval. We find that, as long as the ramp time is sufficiently short (i.e., $\lesssim 0.02 T$ ), very similar results are obtained. Experiments typically use driving frequencies of the order of $\mathrm{kHz}$, which would thus demand ramp times of $\sim 10 \mu \mathrm{s}$, which are achievable.

\section{B. Directed motion}

We can thus see that $\phi$ can be used to cause an initially stationary wave packet to move in a given direction with a precisely defined velocity, without requiring the spatial symmetry of the lattice to be broken. Two values of $K / \omega$ are of particular interest and are marked in Figs. 1(b) and 1(d). For $K / \omega=2.404$ (the first zero of $\mathcal{J}_{0}$ ) the expansion of the initial wave packet is suppressed, and its induced velocity is zero for all values of the driving phase. This amounts to a complete suppression of the dynamics of the condensate. However, at $K / \omega=\pi / 2$ a wave packet that is sinusoidally driven will not expand but will have a nonzero velocity - a directed current of nondispersive wave packets.

In Fig. 2 we show the motion of such a wave packet. Initially we set $K / \omega=\pi / 2$ to induce motion. The driving is then tuned to $K / \omega=2.404$ to bring the wave packet to a halt, and then to $K / \omega=-\pi / 2$ to move the wave packet in the opposite direction. It is clear that the spreading of the wave packet is negligible, and that this technique indeed gives excellent control over the system. It is interesting to note that a similar form of control was reported in Ref. [17] for an amplitude-modulated lattice instead of the phase-modulated lattice we consider. An important difference between the two cases, however, is that phase modulation does not require the presence of a static lattice tilt, since the effects also occur for $n=0$, whereas amplitude modulation is limited to the case of resonant driving $(n>0)$. In addition, amplitude modulation does not produce CDT; the intersite tunneling depending

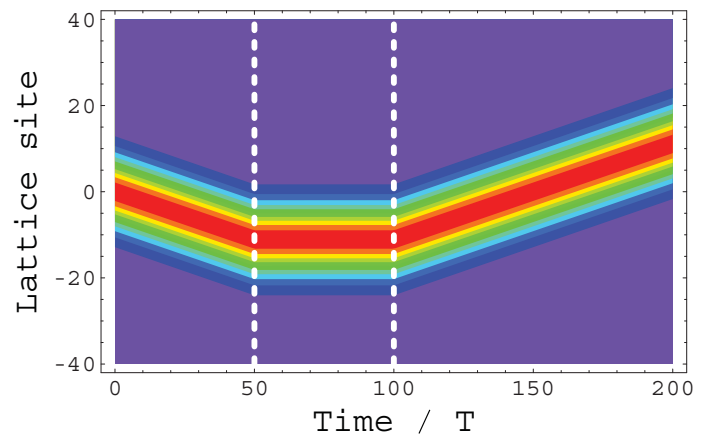

FIG. 2. (Color online) Density plot of the motion of a wave packet under sinusoidal driving. Initially $K / \omega=\pi / 2$ to induce dispersionless transport. $K / \omega$ is then set to 2.404 to freeze the motion and finally to $-\pi / 2$ to reverse it. The vertical dotted lines indicate the times at which $K / \omega$ is changed.

linearly on the driving amplitude instead of the Bessel function dependence given in Eq. (2).

\section{Wave-packet splitting}

We now consider the effect of the onsite phases $\theta_{j}$. It is well known [10] that imprinting a wave packet with a uniform phase gradient $\theta_{j+1}-\theta_{j}=\theta$ has the effect of inducing motion of the center of mass, similar to the motion we have observed by manipulating the driving phase $\phi$. By simulating the system with different values of $\theta$, we have confirmed that the two phases combine, so that the net motion of the wave packet actually depends on the phase difference $\phi-\theta$. The driving field can thus be used to separate components of a wave packet which possess different phase gradients. Let us consider the case of a superposition of a wave packet with uniform phase $(\theta=0)$ and one with a $\pi$ phase $(\theta=\pi)$. If the components have equal weight, the superposition will have the form $\psi_{j}=$ $N \exp \left[-j^{2} /\left(2 \sigma_{0}^{2}\right)\right]$ for $j$ odd and $\psi_{j}=0$ for $j$ even (with no loss of generality we can interchange the roles of the odd and even sites). Such a state can be prepared, for example, by patterned loading of a single uniform-phase condensate.

Under sinusoidal driving for $K / \omega=\pi / 2$, the component with uniform phase will move without distortion at a negative velocity, while the $\pi$-phase component will move identically

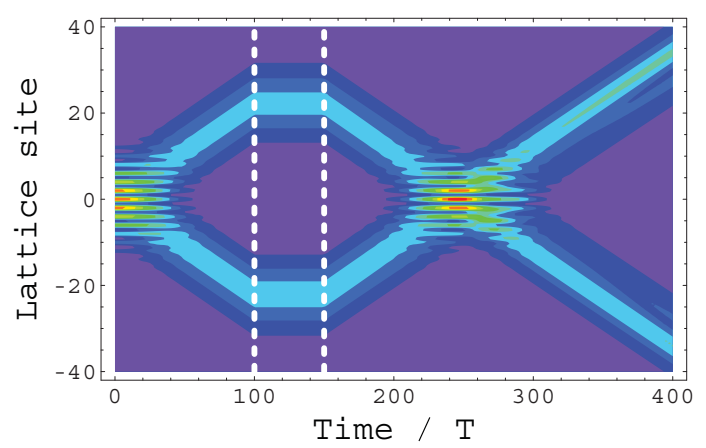

FIG. 3. (Color online) Density plot of a two-component wave packet under sinusoidal driving. Initially $K / \omega=\pi / 2$ and the wave packet splits apart. Its motion is then halted and reversed as before to bring about a collision. The interaction, $g=0.5 \mathrm{~J}$, causes the final state to be asymmetric. 

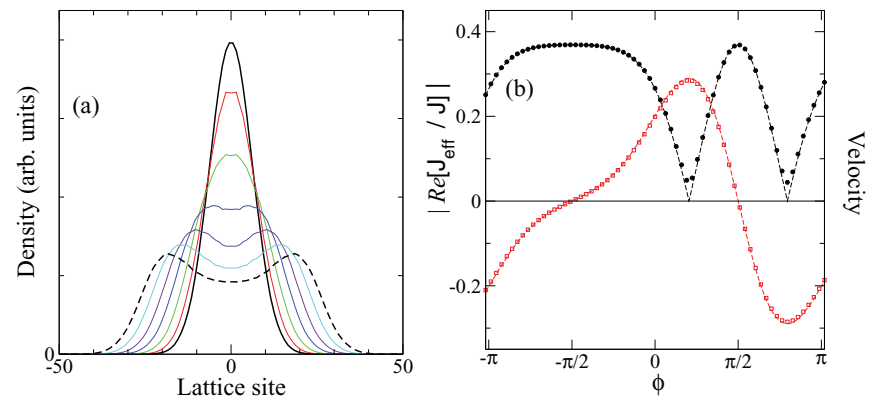

FIG. 4. (Color online) (a) Expansion of a phase-incoherent wave packet, obtained by averaging over 200 random realizations of the onsite phases. Driving parameters are $n=1$ and $K / \omega=0.8$. The density profile is shown in time steps of $24 T$, showing the formation of a double-peak structure as the wave packet spreads. (b) Symbols show results obtained from simulations for the real component of the effective tunneling, $\operatorname{Re}\left[J_{\text {eff }}\right]$ (black circles), which controls the rate of wave-packet spreading, and velocity (red squares), given in units of $d_{L} / T$. Dashed lines show the analytical results. The minimum values of $\operatorname{Re}\left[J_{\text {eff }}\right]$ align with the maximum velocity and vice versa.

but with a positive velocity. The initial wave packet will thus split apart, as shown in Fig. 3. Tuning $K / \omega$ to 2.404 will bring each component to a stop, and then setting $K / \omega=-\pi / 2$ will bring the wave packets together. For zero interaction $(g=0)$ the wave packets will simply pass through each other. For small values of $g$ the splitting process occurs as before but, during the collision the interaction causes the wave packets to distort and produces a slightly asymmetric final state, as shown.

\section{Incoherent expansion}

An intriguing result seen in Ref. [6] is that, unlike previous driven-lattice experiments $[3,4]$, the wave packet deformed under resonant driving, developing pronounced edges during its expansion. As a possible explanation of this effect we now look at the expansion of a phase-incoherent wave packet by averaging over many realizations of random onsite phases $\theta_{j}$. The result in Fig. 4(a) is strikingly similar to the experimental observation.

The phase effects we have discussed give a simple explanation of this behavior. A phase-incoherent wave packet can be expressed as a mixture of many wave packets, each with a random but constant phase gradient $\theta$. Under the periodic driving each component will both develop a certain velocity and spread, according to Eqs. (3) and (4). Because the components that spread least have the highest velocity whereas those that move more slowly spread more rapidly [see Fig. 4(b)], the initial state will segregate with the rapidly moving components at the edges of the wave packet, remaining taller and narrower than the slower-moving components near the center. The edges of the wave packet will move at the maximum speed which, for $n=1$, is given by $v_{\max }=\left|2 \mathcal{J}_{1}(K / \omega)\right|$. For the driving parameters used, our model predicts $v_{\max }=847 d_{L} / \mathrm{s}$, where $d_{L}$ is the lattice spacing. This result compares well with the experimentally measured value of $869 d_{L} / \mathrm{s}$. We thus suggest that the unusual expansion seen in Ref. [6] is a consequence of the phase incoherence of the initial state, possibly arising from phase randomization produced by Wannier-Stark localization during the preparation of the system.

\section{CONCLUSIONS}

We have shown how the phase of a driving potential can be used to control the dynamics of an atomic wave packet, both by regulating its rate of expansion and by inducing a steady drift of its center of mass. Combining these effects allows the directed transport of nondispersive wave packets. Periodic driving also acts as a "prism" for the separation of different phase contributions within a wave packet. This allows wave packets to be divided and recombined and also provides an appealing explanation for the unusual condensate expansion observed in Ref. [6]. While these results have been obtained within mean-field theory, probing the behavior of systems in the strongly correlated regime remains an interesting subject for future research, holding out the enticing prospect of using these effects to generate and distribute entanglement in coherent-lattice systems. We also note that, since these directed currents require coherence across many lattice sites and driving cycles, their eventual decay may provide information on decoherence mechanisms.

\section{ACKNOWLEDGMENTS}

The authors thank Oliver Morsch for many stimulating discussions and acknowledge support from the Spanish MICINN through Grant Nos. FIS2007-65723 and FIS201021372, Acción Integrada HI2008-0163, and the Ramón y Cajal program (CEC).
[1] O. Morsch and M. Oberthaler, Rev. Mod. Phys. 78, 179 (2006).

[2] F. Grossmann, T. Dittrich, P. Jung, and P. Hänggi, Phys. Rev. Lett. 67, 516 (1991).

[3] H. Lignier, C. Sias, D. Ciampini, Y. Singh, A. Zenesini, O. Morsch, and E. Arimondo, Phys. Rev. Lett. 99, 220403 (2007).

[4] C. Sias, H. Lignier, Y. P. Singh, A. Zenesini, D. Ciampini, O. Morsch, and E. Arimondo, Phys. Rev. Lett. 100, 040404 (2008).
[5] A. Alberti, V. V. Ivanov, G. M. Tino, and G. Ferrari, Nature Phys. 5, 547 (2009).

[6] E. Haller, R. Hart, M. J. Mark, J. G. Danzl, L. Reichsöllner, and H.-C. Nägerl, Phys. Rev. Lett. 104, 200403 (2010).

[7] H. Lignier, A. Zenesini, D. Ciampini, O. Morsch, E. Arimondo, S. Montangero, G. Pupillo, and R. Fazio, Phys. Rev. A 79, 041601 (2009).

[8] A. R. Kolovsky, Europhys. Lett. 93, 20003 (2011).

[9] D. Jaksch and P. Zoller, New J. Phys. 5, 56 (2003); F. Gerbier and J. Dalibard, ibid. 12, 033007 (2010). 
[10] J. Denschlag et al., Science 287, 97 (2000).

[11] M. Holthaus, Phys. Rev. Lett. 69, 351 (1992).

[12] D. H. Dunlap and V. M. Kenkre, Phys. Rev. B 34, 3625 (1986).

[13] C. E. Creffield and F. Sols, Phys. Rev. Lett. 100, 250402 (2008).

[14] K. Kudo and T. S. Monteiro, Phys. Rev. A 83, 053627 (2011).
[15] A. Eckardt, M. Holthaus, H. Lignier, A. Zenesini, D. Ciampini, O. Morsch, and E. Arimondo, Phys. Rev. A 79, 013611 (2009); A. Eckardt, C. Weiss, and M. Holthaus, Phys. Rev. Lett. 95, 260404 (2005).

[16] C. E. Creffield, F. Sols, D. Ciampini, O. Morsch, and E. Arimondo, Phys. Rev. A 82, 035601 (2010).

[17] A. Alberti, G. Ferrari, V. V. Ivanov, M. L. Chiofalo, and G. M. Tino, New J. Phys. 12, 065037 (2010). 